\title{
Misalignment Induced Artifacts in Quantitative Annular Bright-Field Imaging
}

\author{
Peng Gao ${ }^{1,2}$, Akihito Kumamoto ${ }^{1}$, Ryo Ishikawa ${ }^{1}$, Nathan Lugg ${ }^{1}$, Naoya Shibata ${ }^{1}$, Yuichi Ikuhara ${ }^{1}$ \\ ${ }^{1}$ Institute of Engineering Innovation, School of Engineering, The University of Tokyo, Tokyo, Japan \\ ${ }^{2}$ Electro Microscopy Laboratory, School of Physics, Center for Nanochemistry, and Collaborative \\ Innovation Center for Quantum Matter, Peking University, Beijing, China
}

Local structural distortion at defects such as grain boundaries, hetero-interfaces, dislocations and surfaces can significantly influence on a broad variety of physical properties in complex oxide materials. Precise measurement of localized structure distortion at these defects can provide new insights into mechanistic understanding as to how materials properties quantitatively depend on the microstructures and subsequently how we can engineer defects to optimize the materials/devices. The recent advances in aberration corrected (scanning) transmission electron microscopy $\left(C_{\mathrm{s}}\right.$-S/TEM) have made it possible to measure the distance between atom positions with picometer-precision [1-3]. In particular, the annular bright field (ABF) imaging [4] allows us to simultaneous visualize both heavy and light element columns over a wide range of thickness, enabling determination of positions of full atomic species from a single image for quantitative measurements.

Here, we study the effects of small crystal tilt on precision measurement of ABF images with an emphasis on calculating and correcting the artifacts and thus avoiding misinterpretation of atomic structures. We choose perovskite $\mathrm{SrTiO}_{3}$ single crystal with perfect cubic structure as a demonstration example (in Figure 1a). A probe forming $C_{\mathrm{s}}$-corrected STEM (JEM-ARM300F; JEOL, Inc.) with spatial resolution up to $\sim 45 \mathrm{pm}$ at $300 \mathrm{kV}$ is employed to simultaneously record both ADF and ABF - STEM images to maximize the precision of measurements. We also have performed systematic multi-slice image simulation for quantitative comparison with experiment in order to correct the specimen tilt effect during data processing.

We find that in the tilted crystal the shift of atom positions depends on atom species, i.e., larger shift for lighter anion columns and smaller shift for heavier cation columns. Under typical convergence semiangle $\sim 24 \mathrm{mrad}$, even with small tilt $\sim 6 \mathrm{mrad}\left(0.34^{\circ}\right)$ and thickness of $25 \mathrm{~nm}$ in $\mathrm{SrTiO}_{3}$ along [001] direction, an artificial displacement $\sim 11.9$ pm between cationic and anionic columns appears (Figure 1b, c, and d), which may lead to significant misinterpretation of microstructure. The same misalignment induced artificial displacement between $\mathrm{Sr}$ and $\mathrm{TiO}$ columns, however, is only about 0.4 pm (Figure 1d). Therefore, in the ABF images the distance measurements between cationic and anionic columns seems more sensitive to the specimen tilt compared to the distance measurements within cationic columns. In other words, the effects of specimen misalignment on precision measurements in the ABF images is more important than that in the ADF images which usually do not show light anion columns. Moreover, the precision of distance measurement between cationic columns in ADF images is believed mainly from the scanning noise and sample drift induced image deformation. However, our results show that the artificial displacement between cationic $\mathrm{Sr}$ and $\mathrm{TiO}$ columns which contains the scan noise, distortion and misalignment, is only about $\sim 3.2 \mathrm{pm}$, indicating the scan noise and distortion induced artificial displacement is also much smaller than that resulted from the misalignments between cationic and anionic columns in the $\mathrm{ABF}$ image.

The tilt induced artificial displacements also depend on the defocus, thickness of specimen and 
convergence angle that is shown in Figure 1e. Under some certain experimental conditions, such artifact can completely dominate the measurement even the tilt is as small as $0.5 \mathrm{mrad}\left(0.03^{\circ}\right)$. Since such small specimen tilt is inevitable on the practical examinations of the crystal with local structural distortion, it is critical to take the tilt effect into account for precise measurement between cationic and anionic columns for ABF images. [5]

\section{References:}

[1] C.L. Jia et al, Nature Materials, 6 (2007) 64.

[2] C.T. Nelson et al, Nano Letters, 11 (2011) 828.

[3] P. Gao et al, Nature Communications, 4 (2013) 2791.

[4] S. Findlay et al, Ultramicroscopy, 110 (2010) 903.

[5] The authors gratefully acknowledge the financial support through a Grant-in-Aid for Scientific Research on Innovative Areas "Nano Informatics" (Grant No. 25106003) from Japan Society for the Promotion of Science (JSPS), and "Nanotechnology Platform" (Project No. 12024046) from the Ministry of Education, Culture, Sports, Science and Technology in Japan (MEXT).
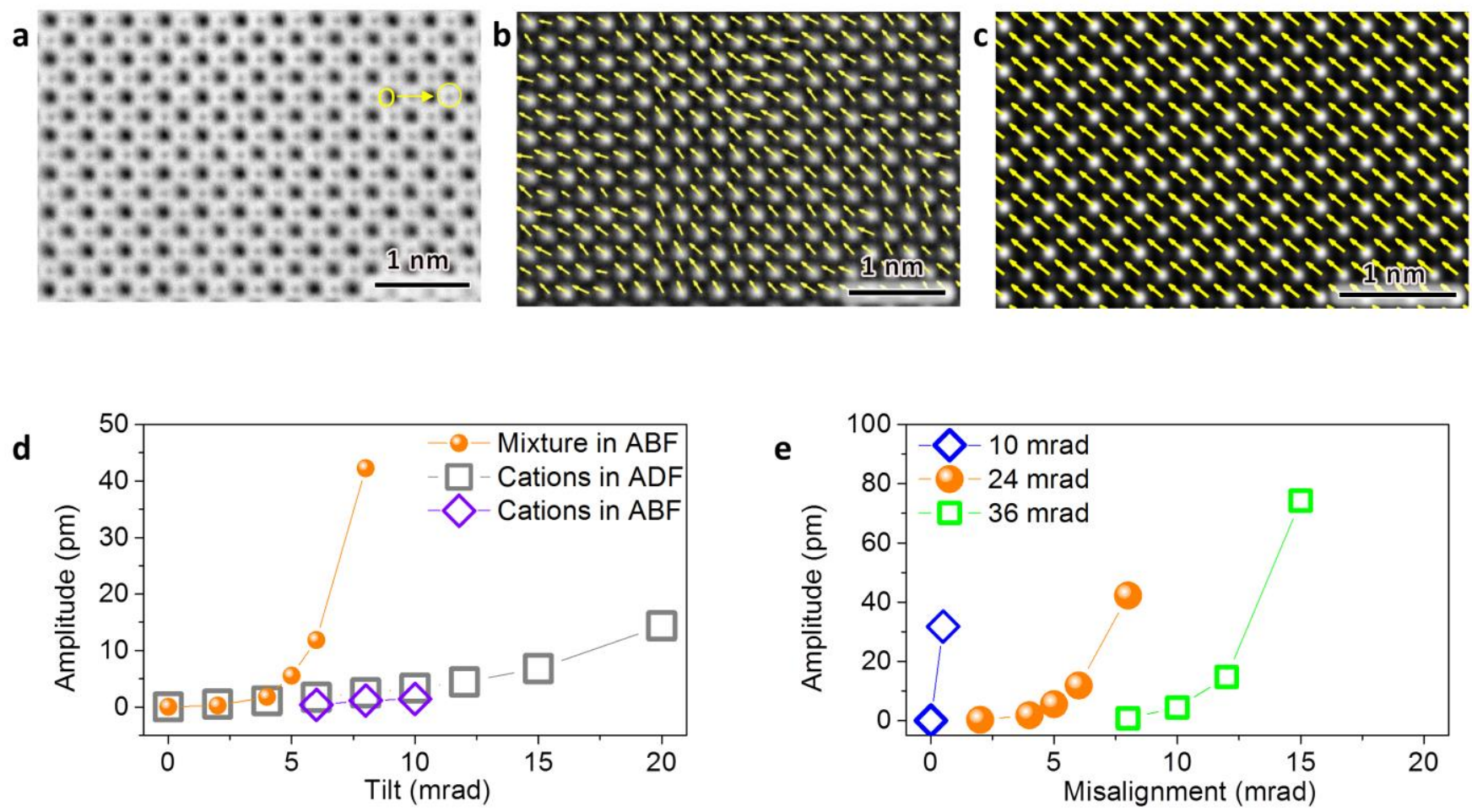

Figure 1. (a) Atomically resolved $\mathrm{ABF}$ image of $\mathrm{SrTiO}_{3}$ single crystal. (b) Map of displacement vectors between $\mathrm{O}$ and $\mathrm{Sr} / \mathrm{TiO}$ columns in the ABF image. The image contrast has been inverted for clarity. (c) Map of displacement vectors in the simulated ABF image with viewing direction of $[0.008,0.005,1]$, thickness of $25 \mathrm{~nm}$, and defocus of $0.5 \mathrm{~nm}$. (d) Amplitude of displacement vectors calculated from the simulated ADF and ABF images. (e) Amplitude of displacement vectors in the simulated ABF images with thickness of $25 \mathrm{~nm}$, defocus of $0 \mathrm{~nm}$ and various convergence and collection angles. Blue diamond: $10 \mathrm{mrad}$ and [5,10] mrad. Orange ball: $24 \mathrm{mrad}$ and [12,24] mrad. Green square: $36 \mathrm{mrad}$ and [18, 36]. 